\title{
Clinical Significance of Trochlear Calcifications in the Orbit
}

\author{
K. Buch, R.N. Nadgir, A.D. Tannenbaum, A. Ozonoff, A. Fujita, and O. Sakai
}

\begin{abstract}
BACKGROUND AND PURPOSE: Trochlear calcification is frequently seen on CT scans that include the orbits, but prior studies linking this finding to diabetes mellitus were based on selected populations by using relatively thick sections. We assessed the relationship between trochlear calcification and diabetes mellitus and other chronic medical conditions in an unrestricted population by using thin-section CT.
\end{abstract}

MATERIALS AND METHODS: Noncontrast head CT studies performed consecutively on 1000 patients between January and February 2011 were retrospectively reviewed for the presence of trochlear calcifications. Axial 1.25-mm-thick images acquired by 64-detector row CT were reviewed. Medical records were reviewed for chronic medical conditions, including diabetes mellitus, chronic kidney disease, alcoholism, autoimmune conditions, endocrine disorders, serum calcium and alkaline phosphatase levels, and HIV status. Motion-limited studies and patients with limited clinical data were excluded. Statistical analyses were performed by using the Fisher exact test.

RESULTS: Thirty-two patients were excluded from analysis. Of the remaining 968 patients, 128 had trochlear calcification (13\%). No significant association was observed between diabetes mellitus and trochlear calcifications in patients of any age $(P=.40)$. There was no significant correlation between trochlear calcifications and chronic kidney disease, alcoholism, endocrine disorders, or HIV infection. Statistically significant associations among trochlear calcification, autoimmune conditions, and elevated alkaline phosphatase levels were observed $(P<.0001$ and $P<.0001$, respectively).

CONCLUSIONS: On the basis of a large unrestricted population with thin-section CT imaging of the orbit, no significant association was observed between trochlear calcifications and diabetes mellitus. A statistically significant association was observed between trochlear calcification and autoimmune disease and elevated alkaline phosphatase levels.

ABBREVIATIONS: $\mathrm{ALP}=$ alkaline phosphatase; $\mathrm{CKD}=$ chronic kidney disease; $\mathrm{DM}=$ diabetes mellitus; $\mathrm{SLE}=$ systemic lupus erythematosus

$\mathbf{T}$ he trochlea is a cartilaginous structure within the superomedial orbit that permits unimpeded movement of the superior oblique tendon and sheath. Incidental calcification of the trochlea is frequently seen on routine CT scans through the orbit. ${ }^{1-8}$ A prior study based on review of dedicated orbital CT imaging linked the presence of trochlear calcification with diabetes mellitus (DM), particularly in patients younger than 40 years of age. ${ }^{1}$ In daily practice, however, review of medical

Received May 1, 2013; accepted after revision June 7.

From the Departments of Radiology (K.B., R.N.N., A.D.T., A.F., O.S.) and Otolaryngology-Head and Neck Surgery (O.S.), Boston Medical Center, Boston University School of Medicine, Boston, Massachusetts; and Biostatistics Core (A.O.), Clinical Research Program, Children's Hospital Boston, Boston, Massachusetts.

Paper previously presented at: 98th Scientific Assembly and Annual Meeting of Radiological Society of North America, November 24-30, 2012; Chicago, Illinois.

Please address correspondence to Osamu Sakai, MD, PhD, Department of Radiology, Boston Medical Center, Boston University School of Medicine, Boston, FGH Building, 3rd Floor, 820 Harrison Ave, Boston, MA 02118; e-mail: osamu.sakai@ bmc.org

http://dx.doi.org/10.3174/ajnr.A3697 records in patients with trochlear calcification incidentally seen on CT usually failed to reveal evidence of glucose intolerance. While Ko and $\mathrm{Kim}^{2}$ did not identify a similar association in a small review of orbital CT examinations, they observed an increased prevalence of trochlear calcifications in male patients. Cartilaginous and soft-tissue calcifications in other anatomic regions have been described in multiple prior studies in patients with autoimmune disorders and chronic renal insufficiency and among patients with dysregulations in calciumphosphatase homeostasis. ${ }^{9}$

The purpose of this study was to examine the prevalence and demographics of trochlear calcification in a large unrestricted patient population by using thin-section CT images and to evaluate its clinical associations, if any. Specifically, we looked for a correlation between the presence of trochlear calcifications and common chronic medical conditions such as $\mathrm{DM}$, chronic kidney disease (CKD), alcoholism, autoimmune conditions, endocrine disorders, and HIV infection. 

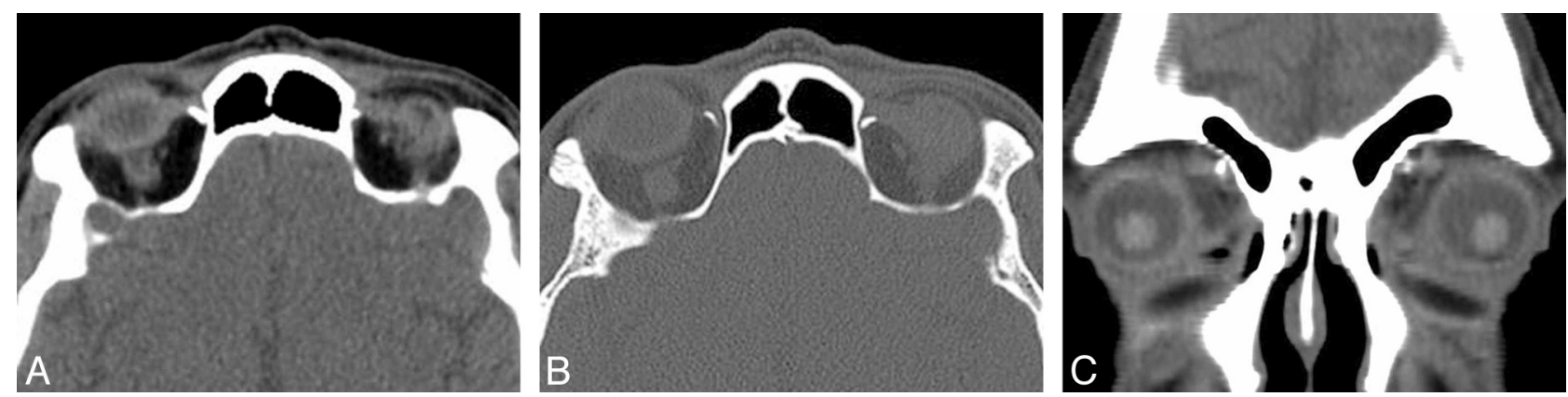

FIG 1. Trochlear calcification. Axial CT images in soft-tissue $(A)$ and bone $(B)$ windows, and coronal CT image in a soft-tissue window $(C)$ through the level of the trochlea demonstrate bilateral trochlear calcifications in a 45-year-old man who underwent CT after a motor vehicle collision and loss of consciousness.

\section{MATERIALS AND METHODS}

Following institutional review board approval, 1000 consecutive patients who underwent a noncontrast head CT at our tertiary health care center between January 2011 and February 2011 were reviewed for the presence of trochlear calcifications, regardless of the clinical indication prompting the CT examination. Reasons for the CT scans were very broad, with the most common clinical indications related to trauma, headache, and altered mental status.

Noncontrast head CT scans were axially acquired on 64-detector row CT scanners (LightSpeed VCT; GE Healthcare, Milwaukee, Wisconsin), and 5- and 1.25-mm-thick images were reconstructed per our institutional clinical protocol. The trochlea was included in all studies. The presence of trochlear calcifications was independently verified by 2 readers on the $1.25-\mathrm{mm}$ axial images, and any discrepancies were resolved by consensus. Note was also made as to whether calcifications were unilateral or bilateral.

The electronic medical records were subsequently reviewed in all patients, and clinical parameters, including age, sex, presence or absence of DM, HIV infection, renal disease, alcoholism, autoimmune disorders, other endocrine disorders, and markers for abnormal calcium-phosphatase homeostasis by using serum markers including alkaline phosphatase (ALP) and serum calcium levels, were recorded. Electronic medical records were reviewed for at least 1 year before the date of the CT scan for the patients included in this study.

CKD was determined on the basis of review of patient medical records documenting a diagnosis of CKD or by using the National Kidney Foundation's clinical practice guidelines for a diagnosis of CKD. ${ }^{10}$ These parameters included abnormal serum creatinine or glomerular filtration rate levels for a period of $>3$ contiguous months. Patients presenting with acute renal failure were not included in this analysis. A diagnosis of alcoholism was determined by a review of the electronic medical records documenting a history of alcohol abuse. Determination of HIV status was made through review of electronic medical records, including serology data in addition to the primary care physician's notes recording a diagnosis of HIV infection. Autoimmune diseases were recorded on the basis of a review of the electronic medical records documenting a diagnosis of a rheumatologic condition in addition to serologic testing data. Endocrine disorders were also determined by a review of the electronic medical records containing a diagnosis of endocrine abnormality and laboratory data examining metabolic markers such as thyroid function tests and calcium and prolactin levels.
Severely motion-limited studies, follow-up examinations if performed in the same period, and patients with insufficient clinical data were excluded. The prevalence of comorbidities was compared across groups by using the Fisher exact test, and logistic regression models were used to control for age and sex.

\section{RESULTS}

A total of 968 patients who underwent technically adequate head CT imaging with corresponding medical records available were included. Eleven patient head CT scans were excluded on the basis of poor image quality due to motion artifacts, and 21 patients were excluded for insufficient electronic medical records. This study population consisted of 456 males and 512 females, ranging in age from 1 month to 100 years (mean age, 53 years). Trochlear calcifications were observed in 128 patients (Fig 1) and were bilateral in 56 patients and unilateral in 72 patients.

\section{Prevalence}

The prevalence of trochlear calcifications was 13.2\% (128/968). The Table displays the prevalence of trochlear calcifications stratified by patient age. There was no association between trochlear calcifications and increasing patient age. Patients 11-20 years of age had the highest prevalence of trochlear calcifications (23.1\%), followed by patients $31-40$ years of age (20.2\%). The youngest patient with trochlear calcifications was 15 years of age. There were 6 patients younger than 15 years of age, none of whom had trochlear calcifications. Trochlear calcifications were slightly more prevalent in male patients, occurring in 75 of the 128 patients with trochlear calcification (58.6\%) compared with $53 \mathrm{fe}$ male patients $(41.4 \%)$. There was no statistically significant correlation between trochlear calcification and sex, however $(P=.06)$.

\section{Diabetes Mellitus}

The prevalence of DM in this study population was $24.1 \%$ (234/ 968). Two hundred twenty-one of 234 patients (94.4\%) had type 2 diabetes, compared with 14 patients (5.6\%) with a diagnosis of type $1 \mathrm{DM}$. Of 128 patients with trochlear calcifications, 26 had a diagnosis of DM, of whom 23 had the diagnosis of DM type 2 and 3 had the diagnosis of DM type 1 . Of the 840 patients without trochlear calcifications, 209 patients had a diagnosis of diabetes. After controlling for age and sex, there was no statistically significant association between DM and trochlear calcification $(P=$ $.27, \mathrm{OR}=0.76$ ). Patient age of 54 years and older, but not patient sex, was strongly associated with the prevalence of DM. 
Prevalence of patients with trochlear calcifications stratified by patient age

\begin{tabular}{lccc} 
Patient Age (yr) & $\begin{array}{c}\text { Total No. } \\
\text { of Patients }\end{array}$ & $\begin{array}{c}\text { Patients with Trochlear } \\
\text { Calcifications }\end{array}$ & $\begin{array}{c}\text { Prevalence of Trochlear } \\
\text { Calcifications (95\% Cl) }\end{array}$ \\
\hline $0-10$ & 23 & 0 & $0.0 \%(-,-)$ \\
$11-20$ & 26 & 6 & $23.1 \%(9.8-44.1)$ \\
$21-30$ & 109 & 17 & $15.6 \%(9.6-24.1)$ \\
$31-40$ & 104 & 21 & $20.2 \%(13.2-29.4)$ \\
$41-50$ & 168 & 24 & $14.3 \%(9.5-20.7)$ \\
$51-60$ & 206 & 25 & $12.1 \%(8.2-17.6)$ \\
$61-70$ & 128 & 15 & $11.7 \%(6.9-18.9)$ \\
$71-80$ & 114 & 9 & $7.8 \%(3.9-14.9)$ \\
$81-90$ & 65 & 9 & $13.8 \%(6.9-25.2)$ \\
Older than 91 & 24 & 2 & $8.3 \%(1.5-28.5)$ \\
Total & 968 & 128 & $13.2 \%(11.2-15.6)$ \\
\hline
\end{tabular}

each type of autoimmune condition in this category, however, determination of significant associations with trochlear calcifications could not be reliably assessed.

\section{Serum Calcium and ALP Levels}

Elevated ALP levels were observed in $21.5 \%$ of patients (209/968). Of the 209 patients with elevated ALP levels, $42 \mathrm{had}$ trochlear calcification. There was a strong statistically significant correlation between trochlear calcification and elevated ALP levels $(P<.0001$, OR $=$

\section{Alcoholism}

The prevalence of documented alcoholism in this study population was $14.5 \%$ (140/968). Of 128 patients with trochlear calcifications, 21 had a diagnosis of alcoholism. After controlling for age and sex, there was no statistically significant correlation between trochlear calcifications and a diagnosis of alcoholism $(P=.50$, $\mathrm{OR}=1.19$ ). Male sex, but not age, was strongly associated with alcoholism.

\section{HIV Infection}

The prevalence of HIV infection in this study population was $3.6 \%$ (35/968). Of the 128 patients with trochlear calcifications, 4 had a diagnosis of HIV infection. After controlling for age and sex, there was no significant association between patients with trochlear calcifications and a diagnosis of HIV infection $(P=1.00$, $\mathrm{OR}=0.76$ ). Male sex, but not age, was strongly associated with a diagnosis of HIV infection $(P=.002)$.

\section{Chronic Kidney Disease}

The most common etiologies of CKD in this study population were hypertensive nephropathy and diabetic nephropathy. The prevalence of CKD in this study population was $8.4 \%$ (81/968). Of 128 patients with trochlear calcifications, 9 had a diagnosis of CKD. After controlling for age and sex, there was no significant association between $\mathrm{CKD}$ and the presence of trochlear calcification $(P=.73$, OR $=0.81)$. Increasing age, but not sex, was strongly associated with CKD $(P=.0001)$.

\section{Autoimmune Diseases}

Autoimmune diseases in this patient population included rheumatoid arthritis, systemic lupus erythematosus (SLE), Crohn disease, Sjögren syndrome, and scleroderma. Forty-eight of 968 patients $(5.0 \%)$ included in this investigation had a history of an autoimmune disease. The most common autoimmune disease identified in this population was rheumatoid arthritis, observed in 22 of 48 patients. Ten patients had SLE, 6 had Crohn disease, 5 had Sjögren syndrome, and 5 had scleroderma. Of the 128 patients with trochlear calcification, 17 had a diagnosis of an autoimmune disease, including 6 with a history of rheumatoid arthritis, 5 with SLE, 3 with Crohn disease, 1 with Sjögren syndrome, and 2 with scleroderma. After we controlled for age and sex, there was a statistically significant association between trochlear calcifications and a history of autoimmune disease $(P<.0001, \mathrm{OR}=$ 3.73). Given the relatively small number of patients included for
1.87). Of the 128 patients with trochlear calcifications, none had elevated serum calcium levels.

\section{Endocrine Disease}

The most common endocrine disorders included in this study (excluding DM, which was analyzed separately) were hyperthyroidism, hypothyroidism, hyperparathyroidism, syndrome of inappropriate antidiuretic hormone, diabetes insipidus, and hyperprolactinemia.

Endocrinopathy other than diabetes was observed in 97 of 968 patients included in this investigation. Of these 97, thirty-eight patients had hypothyroidism, 14 had hyperthyroidism, 3 had acromegaly, 13 had hyperparathyroidism, 16 had hyperprolactinemia, 7 had syndrome of inappropriate antidiuretic hormone, and 6 had diabetes insipidus. Of the 128 patients with trochlear calcification, 10 had a diagnosis of endocrine disease, including 7 patients with hypothyroidism, 2 patients with hyperthyroidism, and 1 patient with acromegaly. No patient with hyperparathyroidism, hyperprolactinemia, syndrome of inappropriate antidiuretic hormone, or diabetes insipidus had trochlear calcifications. After we controlled for age and sex, there was no statistically significant correlation between trochlear calcifications and endocrine diseases $(P=.75, \mathrm{OR}=1.44)$.

\section{DISCUSSION}

Trochlear calcifications have been reported in studies with conflicting data regarding the etiology and clinical association of these calcifications with common medical conditions. ${ }^{1,2}$ Prior studies postulated that calcification of the trochlea may be secondary to degenerative, inflammatory, metabolic, or posttraumatic etiologies. ${ }^{2}$

Hart et $\mathrm{al}^{1}$ described a correlation between trochlear calcifications in patients younger than 40 years of age and DM. ${ }^{1}$ Ko and $\mathrm{Kim}^{2}$ subsequently found no correlation between trochlear calcifications and DM; however, they noted an increased prevalence of trochlear calcifications in male patients. These studies were performed by using thicker section CT images $(3 \mathrm{~mm}$ in the study performed by Hart et al and $2 \mathrm{~mm}$ reported by Ko and Kim) and were performed in small, preselected populations of 159 and 216 patients, respectively, by using dedicated orbital CT studies in patients with orbital symptoms and pathology. ${ }^{1,2}$ Given that orbital symptoms and visual disturbances are common among patients with diabetes, these patient populations may be biased, with 
a higher prevalence of patients with diabetes among the patient population compared with the general population. ${ }^{11,12}$

In this investigation, the patient population consisted of consecutive patients who underwent noncontrast head CT imaging, thereby removing the possibility of bias among patients presenting with specific orbital symptoms. The larger patient population included in this study provides a broader assessment of trochlear calcifications compared with previously published reports. Furthermore, the thinner 1.25 -mm CT sections allowed greater accuracy of trochlear calcification identification.

The prevalence of trochlear calcifications in our study was $13.2 \%$ compared with $12 \%$ cited by Hart et $\mathrm{al}^{1}$ and $16 \%$, by Ko and Kim. ${ }^{2}$ No statistically significant correlation was observed between trochlear calcifications and increasing patient age or sex. A bimodal peak distribution was observed in patients with trochlear calcifications from 11 to 20 years of age and 31-40 years of age. The youngest patient with trochlear calcifications was 15 years of age. These observations imply that the presence of trochlear calcifications is probably not related to a degenerative process and is unlikely to be a congenital variant.

A statistically significant association was seen between a diagnosis of autoimmune disease and trochlear calcifications $(P<$ .0001). The numbers for each type of autoimmune disease included in this study were small so that the evaluation of significant associations between trochlear calcifications and specific autoimmune diseases was limited, so autoimmune diseases were analyzed collectively. Calcification and systemic deposition of calcium salts have been described in multiple autoimmune diseases, including scleroderma, dermatomyositis, rheumatoid arthritis, Sjögren syndrome, and SLE, with a mean age of onset of 33.3 years. ${ }^{13,14}$ The deposition of calcification can be idiopathic, dystrophic, or due to calciphylaxis, and while the pathophysiology is unclear, it is thought to relate to circulating chronic inflammatory mediators. ${ }^{15-27}$

Case reports describe both focal and extensive systemic deposition of calcification in patients with both rheumatoid arthritis and SLE, with calciphylaxis described as a rare complication of both diseases. ${ }^{16-23}$ SLE can lead to CKD, a well-known complication $^{22}$; however, CKD was not independently associated with trochlear calcifications. Widespread soft-tissue calcification in chronic inflammatory autoimmune disorders such as Sjögren syndrome, SLE, and rheumatoid arthritis has been described. ${ }^{20-23,25-27}$ Very few case reports describe focal calcifications within the soft tissues of the head and neck, with the exception of retropharyngeal calcific tendinitis and calcification of the longus colli muscle in a patient with rheumatoid arthritis. ${ }^{28}$ On the basis of the results of this investigation, it appears that in addition to calcification within soft tissues previously described, trochlear calcifications may also be seen as an associated finding in patients with autoimmune conditions.

A statistically significant association between the presence of trochlear calcification and elevated ALP levels $(P<.0001)$ was observed in this investigation. Alkaline phosphatase levels were evaluated in this patient population because they are an indirect marker for bone turnover and metabolism and calcium homeostasis. Increased vascular calcifications have been associated with elevated ALP levels in patients with disturbances in mineral-bone metabolism. ${ }^{29-31}$ Aberrations in ALP levels have been seen in patients with chronic inflammatory and autoimmune diseases. ${ }^{29,30}$ It is unclear as to whether these aberrations are due to circulating inflammatory mediators directly related to the underlying disease process versus abnormal bone-mineral metabolism resulting from glucocorticoid therapy to treat these disorders. ${ }^{9,32,33}$ Most patients with elevated ALP levels did not have a coexistent autoimmune disorder or another condition to explain these levels, but nearly half (46.7\%) of patients with an elevated ALP level and an autoimmune disorder had trochlear calcifications. Despite the statistically significant relationship between the presence of trochlear calcifications and elevated ALP levels, the specific reason for the relationship remains unclear because an isolated elevated ALP level is quite nonspecific.

There was no evidence that trochlear calcification is associated with other common medical conditions, including CKD, HIV infection, endocrine diseases, and history of alcohol abuse.

\section{CONCLUSIONS}

Trochlear calcifications are not uncommon, with a prevalence of $13 \%$ and no sex predilection; they also appear to be acquired, though not necessarily secondary to the aging process. On the basis of review of an unrestricted population undergoing thinsection CT imaging, we found no statistically significant association between the presence of trochlear calcifications and other common medical conditions, including DM, CKD, HIV infection, or a history of alcoholism. We did identify strong associations between the presence of trochlear calcifications and autoimmune disease and elevated ALP levels, but the reason for these associations remains elusive and requires further investigation.

Disclosures: Osamu Sakai_UNRELATED: Royalties: McGraw-Hill Companies.

\section{REFERENCES}

1. Hart BL, Spar JA, Orrison WW Jr. Calcification of the trochlear apparatus of the orbit: $\mathrm{CT}$ appearance and association with diabetes and age. AJR Am J Roentgenol 1992;159:1291-94

2. Ko S, Kim Y. Incidence of calcification of the trochlear apparatus in the orbit. Korean J Ophthalmol 2010;24:1-3

3. Murray JL, Hayman LA, Tang RA, et al. Incidental asymptomatic orbital calcifications. J Neuroophthalmol 1995;15:203-08

4. Helveston EM, Merriam WW, Ellis FD, et al. The trochlea: a study of the anatomy and physiology. Ophthalmology 1982;89:124-33

5. Sobel RK, Goldstein SM. Trochlear calcification: a common entity. Orbit 2012;31:94-96

6. Froula PD, Bartley GB, Garrity JA, et al. The differential diagnosis of orbital calcification as detected on computed tomographic scans. Mayo Clin Proc 1993;68:256-61

7. Xiao TL, Kalariya NM, Yan ZH, et al. Trochlear calcification and intraorbital foreign body in ocular trauma patients. Chin J Traumatol 2009;12:210-13

8. Shriver EM, McKeown CA, Johnson TE. Trochlear calcification mimicking an orbital foreign body. Ophthal Plast Reconstr Surg 2011;27:e143-44

9. Nanke $Y$, Kotake S, Akama H, et al. Alkaline phosphatase in rheumatoid arthritis patients: possible contribution of bone-type ALP to the raised activities of ALP in rheumatoid arthritis patients. Clin Rheumatol 2002;21:198-202

10. Kidney Disease Outcome Quality Initiative: Clinical Practice Guidelines for Chronic Kidney Disease: Evaluation, Classification, and Stratification. National Kidney Foundation. Published January 2002. 
Updated April 19, 2003. http://www.kidney.org/professionals/kdoqi/ guidelines_ckd/toc.htm. Accessed July 14, 2013

11. Goldacre MJ, Wotton CJ, Keenan TD. Risk of selected eye diseases in people admitted to hospital for hypertension or diabetes mellitus: record linkage studies. Br J Ophthalmol 2012;96:872-76

12. Jeganathan VS, Wang JJ, Wong TY. Ocular associations of diabetes other than diabetic retinopathy. Diabetes Care 2008;31:1905-12

13. Okada J, Nomura M, Shirataka M, et al. Prevalence of soft tissue calcifications in patients with SLE and effects of alfacarcidol. Lupus 1999;8:456-61

14. Fairweather D, Frisancho-Kiss S, Rose N. Sex differences in autoimmune disease from a pathological perspective. Am J Pathol 2008;173:600-09

15. Shantsila E, Lip GY. Systemic inflammation as a driver of vascular calcification: a proof of concept. J Intern Med 2009;266:453-56

16. Masuda Y, Uchiyama Y, Hashimoto S, et al. Symmetrica 1 progressive intracranial calcification in a patient with SLE. Intern Med 2010;49:351

17. Yiu KH, Mok MY, Wang S, et al. Prognostic role of coronary calcification in patients with rheumatoid arthritis and systemic lupus erythematosus. Clin Exp Rheumatol 2012;30:345-50

18. Plazak W, Pasowicz M, Kostkiewicz M, et al. Influence of chronic inflammation and autoimmunity on coronary calcifications and myocardial perfusion defects in systemic lupus erythematosus patients. Inflamm Res 2011;60:973-80

19. Paccou J, Brazier M, Mentaverri R, et al. Vascular calcification in rheumatoid arthritis: prevalence, pathophysiological aspects and potential targets. Atherosclerosis 2012;224:283-90

20. Llamas-Velasco M, Eguren C, Santiago D, et al. Calcinosis cutis and Sjögren's syndrome. Lupus 2010;19:762-64

21. Ortiz A, Ceccato F, Roverano S, et al. Calciphylaxis associated with rheumatoid arthritis: communication of the second case. Clin Rheumatol 2009;28(suppl 1):S65-68

22. Igaki N, Moriguchi R, Hirota $\mathrm{Y}$, et al. Calciphylaxis in a patient with end-stage renal disease secondary to systemic lupus erythematosus associated with acral gangrene and mesenteric ischemia. Intern Med 2001;40:1232-37

23. Sugimoto $\mathrm{H}$, Hyodoh K, Kikuno M, et al. Periarticular calcification in systemic lupus erythematosus. J Rheumatol 1999;26:574-79

24. MacLean C, Brahn E. Systemic lupus erythematosus: calciphylaxis induced cardiomyopathy. J Rheumatol 1995;22:177-79

25. Minami A, Suda K, Kaneda K, et al. Extensive subcutaneous calcification of the forearm in systemic lupus erythematosus. J Hand Surg Br 1994;19:638-41

26. Mandelbrot DA, Santos PW, Burt RK, et al. Resolution of SLE-related soft-tissue calcification following haematopoietic stem cell transplantation. Nephrol Dial Transplant 2008;23:2679-84

27. Tristano AG, Villarroel JL, Rodríguez MA, et al. Calcinosis cutis universalis in a patient with systemic lupus erythematosus. Clin Rheumatol 2006;25:70-74

28. Lee S, Joo KB, Lee KH, et al. Acute retropharyngeal calcific tendinitis in an unusual location: a case report in a patient with rheumatoid arthritis and atlantoaxial subluxation. Korean $J \mathrm{Ra}$ diol 2011;12:504-09

29. Kapustin AN, Davies JD, Reynolds JL, et al. Calcium regulates key components of vascular smooth muscle cell-derived matrix vesicles to enhance mineralization. Circ Res 2011;109:e1-12

30. Osorio A, Ortega E, Torres JM, et al. Biochemical markers of vascular calcification in elderly hemodialysis patients. Mol Cell Biochem 2013;374:212-17

31. Shroff RC, McNair R, Skepper JN, et al. Chronic mineral dysregulation promotes vascular smooth muscle cell adaptation and extracellular matrix calcification. J Am Soc Nephrol 2010;21:103-12

32. Jensen T, Hansen M, Madsen JC, et al. Serum levels of parathyroid hormone and markers of bone metabolism in patients with rheumatoid arthritis. Relationship to disease activity and glucocorticoid treatment. Scand J Clin Lab Invest 2001;61:491-501

33. Korczowska I, Łacki JK, Leszczyński P, et al. Bone tissue metabolism in systematic lupus erythematosus patients treated with glucocorticosteroids [in Polish]. Pol Arch Med Wewn 2001;106:901-07 